\title{
Antimicrobial resistance in pathogens causing urinary tract infections in and around a tertiary care hospital in south Kerala, India
}

\author{
L Suresh Babu ${ }^{1 *}$, S R Jobin ${ }^{2}$ \\ ${ }^{1}$ Professor, ${ }^{2}$ Assistant Professor, Department of Microbiology, Dr Somervell Memorial CSI Medical College \& Hospital, Karakonam, \\ Trivandrum District, Kerala, Pincode- 695504, INDIA. \\ Email: sureshbabu6831@yahoo.in
}

Abstract Background: Urinary tract infection is the second most common community acquired infection next to respiratory infections. There are few reports of UTI elsewhere in India, but, it is very little in Kerala, that too in the rural areas of Trivandrum district, there is no report covering the antibiotic resistance pattern of the common UTI isolates. So, we have undertaken this study, so as to understand the ever increasing antibiotic resistance pattern of common UTI pathogens in this part of the country. Urine samples were collected between a period of 1-1-2018 and 13-2 2018. Out of the 1000 samples collected during this period, we have isolated $168(16.8 \%)$ pathogens, predominantly bacterial and few fungal agents, from the corresponding 168 UTI cases. Out of these 168 UTI cases, $40(23.8 \%)$ were male and $128(76.2 \%)$ were female. Outpatients $(54.8 \%)$ predominated over the inpatients (45.2\%). Among the uropathogens isolated, Escherichia coli was the dominant (69.6\%) organism followed by Klebsiella spp. (9.5\%), with very few isolates of Enterococcus spp., Staphylococcus aureus, Candida spp., Acinetobacter spp, Pseudomonas spp., Proteus mirabilis and Citrobacter spp, in that order. E. coli being the most predominant isolate, showed increased resistance to Amoxiclav( $85.5 \%$ of isolates), followed by Nalidixic acid (75.3\%), Amoxicillin (73.5\%,), Cefuroxime (54.7\%), Cefixime (53.8\%), Cefotaxime (52.2\%), Ciprofloxacin (46.2\%) and Norfloxacin (40.2\%). Similarly, Klebsiella spp., the second most isolates, reported higher level of resistance to Amoxiclav (87.5\% of isolates), followed by Amoxicillin (56.3\%), Cefuroxime (43.7\%), Cefixime (37.5\%) and Co-trimoxazole (37.5\%). Among the few isolates of Enterococcus spp., the strains exhibited maximum resistance to Norfloxacin (75\% of strains) followed by Penicillin (41.7\%). In Staphylococcus aureus isolates, 50\% of them showed resistance to Penicillin, but no MRSA recorded. Seven multi-drug resistant strains, 4 of E. coli, 2 of Klebsiella spp. and one of Acinetobacter sp., were also seen.

Key words: E. coli, Enterococcus spp., Klebsiella spp., Multi-drug resistant, Trivandrum district, UTI.

\section{*Address for Correspondence:}

Dr L Suresh Babu, Professor, Department of Microbiology, Dr Somervell Memorial CSI Medical College \& Hospital, Karakonam, Trivandrum District, Kerala, Pincode- 695504, INDIA.

Email: sureshbabu6831@yahoo.in

Received Date: 21/12/2019 Revised Date: 13/01/2020 Accepted Date: 04/02/2020

DOI: https://doi.org/10.26611/10081421

\begin{tabular}{|l|l|}
\hline \multicolumn{2}{|c|}{ Access this article online } \\
\hline Quick Response Code: & Website: \\
\hline & www.medpulse.in \\
& \\
\hline
\end{tabular}

\section{INTRODUCTION}

Urinary tract infections (UTIs) are one of the commonly encountered diseases in developing countries with an estimated annual global incidence of at least 250 million. ${ }^{1}$ Although UTIs occur in both men and women, clinical studies suggest that the overall prevalence of UTI is higher in women. Uncomplicated UTIs in healthy women have an incidence of 50 individuals among 1000 women in a year. ${ }^{2}$ Almost $95 \%$ of cases of UTIs are caused by bacteria. ${ }^{3} \mathrm{Up}$ to $85 \%$ of UTIs are caused by Escherichia coli. ${ }^{4}$ Excessive and/or inappropriate use of antibiotics in treating UTIs is responsible for the emergence and spread of multi-drug resistant (MDR) urinary bacteria ${ }^{5}$. There are three mechanisms that can cause antibiotic resistance. Prevention of the interaction of the drug with target organisms, decreased uptake due to either an increased efflux or a decreased influx of the antimicrobial agent, and enzymatic modification or destruction of the compound ${ }^{6}$. 
There were few reports of the resistance rate of uropathogenic E. coli to various antibiotics are available elsewhere in India. ${ }^{7,8,10}$ But, the resistance pattern of community acquired uropathogens has not been extensively studied in the Indian subcontinent. ${ }^{11,12,13}$ There was one report of antibiotic susceptibility pattern of uropathogens recorded in north Kerala ${ }^{14}$, but, none were so far available in South Kerala, that too in rural areas of this region. By keeping all these in mind, we have undertaken this work to study in detail the ever increasing trend in the resistance of uropathogens against common antibiotics, in the rural areas of southern part of Trivandrum district, Kerala.

\section{MATERIALS AND METHODS}

The work has been carried out in the department of Microbiology, Dr Somervell Memorial CSI Medical College and Hospital, Karakonam, Trivandrum district, Kerala, India. A total of 1000 midstream urine samples from suspected cases of urinary tract infections were collected in pre-sterilized disposable universal containers, during a period between 1-1-2018 and 13-2-2018 at the microbiology diagnostic laboratory, Dr SM CSI Medical College and Hospital. The samples were processed within $1 \mathrm{hr}$ of collection as per the standard procedures. A wet mount with the deposit was done first to look for pus cells and/or bacteria, followed culture on to $5 \%$ sheep blood agar and MacConkey agar, purchased from HiMedia, Mumbai, by the standard semi-quantitative calibration loop technique making use of $0.001 \mathrm{ml}$ diameter loop. The plates were incubated at $37^{\circ} \mathrm{C}$ overnight and looked for growth. Those samples yielded 100 colonies or more $\left(10^{5}\right.$ $\mathrm{CFU} / \mathrm{ml}$ of urine) in both the media were considered as showing significant bacteriuria for ascertaining definite UTI. ${ }^{15,16}$ Out of the total 1000 urine samples processed, $168(16.8 \%)$ showed significant bacteriuria and hence were considered as definite UTI cases. Of these 168 UTI cases, $92(54.8 \%)$ were from OPDs, $45(26.8 \%)$ from peripheral multi-speciality hospitals and remaining cases were from intensive care units and other medical wards (Table-5). The organisms isolated from these cases were identified by their colony and biochemical characteristics. $117(69.4 \%)$ of the isolates were Escherichia coli, 16 $(9.5 \%)$ belonged to Klebsiella spp., followed by Enterococcus spp., 12 (7.1\%), Staphylococcus aureus, 6 (3.5\%), Candida spp., 5 (2.9\%), Acinetobacter spp., 4 (2.4\%), $3(1.8 \%)$ each of Pseudomonas spp and Proteus mirabilis and $2(1.2 \%)$ of Citrobacter spp., (Table-6). All the 168 isolates were subjected to antibiotic susceptibility testing by Kirby- Bauer disc diffusion method in MuellerHinton agar, except, Enterococcus isolates, for which it was done in $5 \%$ sheep blood agar, based on the CLSI guidelines. ATCC strains one each of the isolates of bacterial pathogens were employed as quality control. ${ }^{17}$ The antibiotic discs used were, Ampicillin (10 mcg), Amikacin (30 mcg), Amoxicillin+Clavulanic acid (30 $\mathrm{mcg}$ ), Chloramphenicol (30 mcg), Ciprofloxacin (5 mcg), Cloxacillin (200 mcg), Co-trimoxazole $(25 \mathrm{mcg})$, Cephotaxime (30 mcg), Cefuroxime $(30 \mathrm{mcg})$, Cefaclor (30 $\mathrm{mcg})$, Cefixime $(30 \mathrm{mcg})$, Cefazolin $(30 \mathrm{mcg})$, Gentamicin (10 mcg), Linezolid (10 mcg), Meropenem (10 $\mathrm{mcg}$ ), Nofloxacin $(10 \mathrm{mcg})$, Nalidixic acid $(30 \mathrm{mcg})$, Penicillin (10 units), Piperacillin+ Tazobactam (100/10 mcg), Rifampicin (5 mcg), Tobramycin $(10 \mathrm{mcg})$ and Vancomycin (30 mcg), procured from HiMedia, Mumbai. The plates were incubated at $37 \mathrm{oC}$ overnight for every isolate then and there, and the zone inhibition was measured in millimetres to ascertain, whether a particular antibiotic is sensitive or resistant to an isolate, by interpreting from the chart supplied by the disc manufacturer (HiMedia, Mumbai).

\section{OBSERVATIONS AND RESULTS}

Out of the 168 UTI cases, 40 (23.8\%) were male and 128 $(76.2 \%)$ were female [Table-1]. The age group of 31-40 and 61-70 showed the highest distribution of cases of 29 (17.2\%) each, followed by 21-30 of 27 (16\%), 51-60 and $>70$ groups had $23(13.6 \%)$ cases each [Table-2]. Among male, $>70$ age group were the most infected UTI cases, of $13(32.5 \%)$, [Table-3] and in female, 21-30 age group had the highest UTI cases of 27 (21\%), followed by 31-40 having 23 (17.9\%) cases, [Table-4]. Out patients, out numbered the in-patients among the UTI cases, having 92 (54.8\%), followed by peripheral health care centres, 45 (26.8\%), female medical ward, $15(8.9 \%)$ and few cases distributed among intensive health care units [Table-5]. Out of the total 168 confirmed UTI cases, Escherichia coli was the most predominant isolate from 117 (69.6\%) cases, followed by Klebsiella spp., 16 (9.5\%), Enterococcus spp., $12(7.1 \%)$, Staphylococcus aureus, 6 (3.5\%), Candida spp., 5 (2.9\%), Acinetobacter spp., 4 (2.4\%), Pseudomonas spp., and Proteus mirabilis $3(1.8 \%)$ each and Citrobacter spp., $2(1.2 \%)$, [Table-6]. Escherichia coli being the predominant isolate, showed the highest level of resistance to Ampicillin (93.2\%), followed by Amoxiclav (85.5\%), Nalidixic acid (75.3\%), Amoxicillin (73.5\%), Cefuroxime (54.7\%), Cefixime (53.8\%), Cefotaxime (52.2\%), Ciprofloxacin (46.2\%) and Norfloxacin (40.2\%). Meropenem remained the most sensitive drug (98.3\%), followed by Piperacillin+Tazobactam (95.7\%), Amikacin (84.7\%), Gentamicin (73.5\%) and Tobramycin (72.6\%), [Table-7, Figure-1]. Among Klebsiella spp., Ampicillin and Amoxiclav were the most resistant antibiotics (87.5\%), followed by Amoxicillin (56.3\%), Cefuroxime (43.7\%) and Cefixime and Co-trimoxazole, $37.5 \%$ each. Meropenem, Gentamicin and Amikacin were the most 
sensitive drugs (87.5\%), followed by Piperacillin+Tazobactam (81.3\%), [Table-8, Figure-2]. As, there were only 4 isolates of Acinetobacter spp., 3 each of Pseudomonas spp., and Proteus mirabilis and 2 of Citrobacter spp., their sensitivity/resistance pattern is tabulated as number of isolates of each species, sensitive or resistant [Table-9]. Among the gram positive isolates, Enterococcus spp. (12 isolates), recorded resistance to $75 \%$ of the isolates for Norfloxacin, followed by Penicillin (41.7\%). All isolates were sensitive to Vancomycin and Linezolid (100\%), with Chloramphenicol (91.7\%) and Ampicillin (83.3\%) coming close [Table-10, Figure-3]. Of the 6 Staphylococcus aureus isolates, there were no MRSA strains. Only Penicillin showed resistance to $50 \%$ of the strains. All the strains were sensitive to Clindamycin, Cloxacillin, Gentamicin, Linezolid, Rifampicin and Vancomycin (100\%), with others, Norfloxacin, Cefazolin and Co-trimoxazole showing susceptibility to $83.3 \%$ of the isolates [Table-11, Figure-4]. There were also 7 multi-drug resistant isolates, of 4 from E. coli, 2 of Klebsiella spp. and 1 was Acinetobacter sp. Four isolates of Candida spp., other than C. albicans and 1 Candida albicans were also isolated [Table-6].

Table 1: Sex wise distribution of UTI cases $(n=168) 6$

\begin{tabular}{ccc}
\hline Sex & Male & Female \\
\hline No. of UTI cases & 40 & 128 \\
$\%$ of UTI cases & 23.8 & 76.2 \\
\hline
\end{tabular}

Table 2: Age wise distribution of UTI cases ( $n-168$ )

\begin{tabular}{|c|c|c|c|c|c|c|c|c|}
\hline Age group & $0-10$ & $11-20$ & $21-30$ & $31-40$ & $41-50$ & $51-60$ & $61-70$ & $>70$ \\
\hline No. of cases & 13 & 03 & 27 & 29 & 21 & 23 & 29 & 23 \\
\hline$\%$ of cases & 7.7 & 1.7 & 16 & 17.2 & 12.5 & 13.6 & 17.2 & 13.6 \\
\hline Age group & $0-10$ & $11-20$ & $21-30$ & $31-40$ & $41-50$ & $51-60$ & $61-70$ & $>70$ \\
\hline No. of cases & 05 & 00 & 00 & 06 & 06 & 01 & 09 & 13 \\
\hline$\%$ of cases & 12.5 & 00 & 00 & 15 & 15 & 2.5 & 22.5 & 32.5 \\
\hline Age group & $0-10$ & $11-20$ & $21-30$ & $31-40$ & $41-50$ & $51-60$ & $61-70$ & $>70$ \\
\hline No. of cases & 08 & 03 & 27 & 23 & 15 & 22 & 20 & 10 \\
\hline$\%$ of cases & 6.25 & 2.3 & 21 & 17.9 & 11.7 & 17.1 & 15.6 & 7.8 \\
\hline
\end{tabular}

Table 5: Ward wise distribution of UTI cases $(n=168) 7$

\begin{tabular}{ccccccccccccc}
\hline Ward & KK & OP & MICU & PICU & PdW & ANW & FOW & MMW & FMW & MSW & FSW & SICU \\
\hline No. of cases & 45 & 92 & 03 & 01 & 02 & 02 & 01 & 04 & 15 & 01 & 01 & 01 \\
\% of cases & 26.78 & 54.76 & 1.78 & 0.59 & 1.19 & 1.19 & 0.59 & 2.38 & 8.92 & 0.59 & 0.59 & 0.59 \\
\hline
\end{tabular}

KK- Peripheral multispeciality hospitals, OP- Out patient, MICU- Medical intensive care unit, PICU- Paediatric intensive care unit, PdWPaediatric ward, ANW- Antenatal ward, FOW- Female obstetric ward, MMW- Male medical ward, FMW- Female medical ward, MSW- Male surgical ward, FSW- Female surgical ward, SICU- Surgical intensive care unit

\begin{tabular}{ccc} 
Table 6: Prevalence of pathogens among UTI cases $(\mathrm{n}=168)$ \\
\hline Pathogen & No. of cases & \% of cases \\
\hline Escherichia coli & 117 & 69.6 \\
Klebsiella spp. & 16 & 9.5 \\
Acinetobacter spp. & 04 & 2.4 \\
Pseudomonas spp. & 03 & 1.8 \\
Proteus mirabilis & 03 & 1.8 \\
Citrobacter spp. & 02 & 1.2 \\
Enterococcus spp. & 12 & 7.1 \\
Staphylococcus aureus & 06 & 3.5 \\
Candida spp. & 05 & 2.9 \\
\hline
\end{tabular}


Table 7: Antibiotic susceptibility pattern of Escherichia coli isolates ( $n=117$ )

\begin{tabular}{|c|c|c|}
\hline Antibiotic & No. of isolates & $\%$ of isolates \\
\hline Ampicillin S & 08 & 6.8 \\
\hline $\mathrm{R}$ & 109 & 93.2 \\
\hline Amikacin S & 99 & 84.7 \\
\hline $\mathrm{R}$ & 18 & 15.3 \\
\hline Amoxicillin+S & 17 & 14.5 \\
\hline Clavulanic acid R & 100 & 85.5 \\
\hline Amoxicillin S & 31 & 26.5 \\
\hline $\mathrm{R}$ & 86 & 73.5 \\
\hline Ciprofloxacin S & 63 & 53.8 \\
\hline $\mathrm{R}$ & 54 & 46.2 \\
\hline Co-trimoxazole S & 69 & 58.9 \\
\hline $\mathrm{R}$ & 48 & 41.1 \\
\hline Cefotaxime S & 56 & 47.8 \\
\hline $\mathrm{R}$ & 61 & 52.2 \\
\hline Cefuroxime S & 53 & 45.3 \\
\hline $\mathrm{R}$ & 64 & 54.7 \\
\hline Cefaclor S & 77 & 65.8 \\
\hline $\mathrm{R}$ & 40 & 34.2 \\
\hline Cefixime S & 54 & 46.2 \\
\hline $\mathrm{R}$ & 63 & 53.8 \\
\hline Gentamicin S & 86 & 73.5 \\
\hline $\mathrm{R}$ & 31 & 26.5 \\
\hline Meropenem S & 115 & 98.3 \\
\hline $\mathrm{R}$ & 02 & 1.7 \\
\hline Norfloxacin S & 70 & 59.8 \\
\hline $\mathrm{R}$ & 47 & 40.2 \\
\hline Nalidixic acid S & 29 & 24.7 \\
\hline $\mathrm{R}$ & 88 & 75.3 \\
\hline Piperacillin + S Tazobactam R & 112 & 95.7 \\
\hline & 05 & 4.3 \\
\hline Tobramycin S & 85 & 72.6 \\
\hline $\mathrm{R}$ & 32 & 27.4 \\
\hline
\end{tabular}

Table 8: Antibiotic susceptibility pattern of Klebsiella spp. isolates $(n=16)$

\begin{tabular}{ccc}
\hline Antibiotic & No. of isolates & \% of isolates \\
\hline Ampicillin S & 02 & 12.5 \\
R & 14 & 87.5 \\
Amikacin S & 14 & 87.5 \\
R & 02 & 12.5 \\
Amoxicillin + S & 02 & 12.5 \\
Clavulanic acid R & 14 & 87.5 \\
Amoxicillin S & 07 & 43.7 \\
R & 09 & 56.3 \\
Ciprofloxacin S & 11 & 68.7 \\
R & 05 & 31.3 \\
Co-trimoxazole S & 10 & 62.5 \\
R & 06 & 37.5 \\
Cefotaxime S & 11 & 68.7 \\
R & 05 & 31.3 \\
Cefuroxime S & 09 & 56.3 \\
R & 07 & 43.7 \\
Cefaclor S & 11 & 68.7 \\
R & 05 & 31.3 \\
Cefixime S & 10 & 62.5 \\
R & 06 & 37.5 \\
\hline
\end{tabular}




\begin{tabular}{ccc}
\hline Gentamicin S & 14 & 87.5 \\
R & 02 & 12.5 \\
Meropenem S & 14 & 87.5 \\
R & 02 & 12.5 \\
Norfloxacin S & 11 & 68.7 \\
R & 05 & 31.3 \\
Nalidixic acid S & 10 & 62.5 \\
R & 06 & 37.5 \\
Piperacillin + S Tazobactam R & 13 & 81.3 \\
Tobramycin S & 03 & 18.7 \\
R & 11 & 68.7 \\
\multicolumn{2}{c}{ S- sensitive; R- Resistant } \\
\hline
\end{tabular}

Table 9: Antibiotic susceptibility pattern of Acinetbacter spp., Pseudomonas spp., Proteus mirabilis and Citrobacter spp

\begin{tabular}{|c|c|c|c|c|}
\hline Antibiotic & Acinetobacter spp. $(n=4)$ & Pseudomonas spp. $(n=3)$ & Proteus mirabilis $(n=3)$ & Citrobacter spp. $(n=2)$ \\
\hline Ampicillin S & 00 & 00 & 02 & 02 \\
\hline $\mathrm{R}$ & 04 & 03 & 01 & 00 \\
\hline Amikacin S & 00 & 02 & 03 & 02 \\
\hline $\mathrm{R}$ & 04 & 01 & 00 & 00 \\
\hline Amoxicillin+S & 00 & 01 & 03 & 01 \\
\hline Clavulanic acid $\mathrm{R}$ & 04 & 02 & 00 & 01 \\
\hline Amoxicillin S & 00 & 00 & 03 & 01 \\
\hline $\mathrm{R}$ & 04 & 03 & 00 & 01 \\
\hline Ciprofloxacin S & 02 & 02 & 02 & 02 \\
\hline $\mathrm{R}$ & 02 & 01 & 01 & 00 \\
\hline Co- trimoxazole S & 04 & 02 & 03 & 01 \\
\hline $\mathrm{R}$ & 00 & 01 & 00 & 01 \\
\hline Cefotaxime S & 00 & 03 & 03 & 02 \\
\hline $\mathrm{R}$ & 04 & 00 & 00 & 00 \\
\hline Cefuroxime S & 01 & 03 & 03 & 02 \\
\hline $\mathrm{R}$ & 03 & 00 & 00 & 00 \\
\hline Cefaclor S & 04 & 03 & 03 & 02 \\
\hline $\mathrm{R}$ & 00 & 00 & 00 & 00 \\
\hline Cefixime S & 00 & 03 & 03 & 02 \\
\hline $\mathrm{R}$ & 04 & 00 & 00 & 00 \\
\hline Gentamicin S & 02 & 03 & 03 & 00 \\
\hline $\mathrm{R}$ & 02 & 00 & 00 & 02 \\
\hline Meropenem S & 02 & 03 & 03 & 02 \\
\hline $\mathrm{R}$ & 02 & 00 & 00 & 00 \\
\hline Norfloxacin S & 04 & 03 & 03 & 01 \\
\hline $\mathrm{R}$ & 00 & 00 & 00 & 01 \\
\hline Nalidixic acid S & 02 & 02 & 02 & 01 \\
\hline $\mathrm{R}$ & 02 & 01 & 01 & 01 \\
\hline $\begin{array}{l}\text { Piperacillin + S } \\
\text { Tazobactam }\end{array}$ & 04 & 03 & 03 & 02 \\
\hline $\mathrm{R}$ & 00 & 00 & 00 & 00 \\
\hline Tobramycin S & 02 & 03 & 03 & 02 \\
\hline $\mathrm{R}$ & 02 & 00 & 00 & 00 \\
\hline
\end{tabular}

S- sensitive; R-resistant 11

Table 10: Antibiotic susceptibility pattern of Enterococcus spp. isolates ( $n=12$ )

\begin{tabular}{ccc}
\hline Antibiotic & No. of isolates & \% of isolates \\
\hline Ampicillin S & 10 & 83.3 \\
R & 02 & 16.7 \\
Chloramphenicol S & 11 & 91.7 \\
R & 01 & 8.3 \\
Linezolid S & 12 & 100 \\
R & 00 & 00 \\
\hline
\end{tabular}




\begin{tabular}{ccc}
\hline Norfloxacin S & 03 & 25 \\
R & 09 & 75 \\
Penicillin S & 07 & 58.3 \\
R & 05 & 41.7 \\
Vancomycin S & 12 & 100 \\
R & 00 & 00 \\
\hline
\end{tabular}

S- sensitive, R-resistant

Table 11: Antibiotic susceptibility pattern of Staphylococcus aureus isolates ( $n=6)$

\begin{tabular}{ccc}
\hline Antibiotic & No. of isolates & $\%$ of isolates \\
\hline Clindamycin S & 06 & 100 \\
R & 00 & 00 \\
Co-trimoxazole S & 05 & 83.3 \\
R & 01 & 16.7 \\
Cloxacillin S & 06 & 100 \\
R & 00 & 00 \\
Cefazolin S & 05 & 83.3 \\
R & 01 & 16.7 \\
Gentamicin S & 06 & 100 \\
R & 00 & 00 \\
Linezolid S & 06 & 100 \\
R & 00 & 00 \\
Norfloxacin S & 05 & 83.3 \\
R & 01 & 16.7 \\
Penicillin S & 03 & 50 \\
R & 03 & 50 \\
Rifampicin S & 06 & 100 \\
R & 00 & 00 \\
Vancomycin S & 06 & 100 \\
R & 00 & 00 \\
\hline S-sensitive & R-resistant &
\end{tabular}
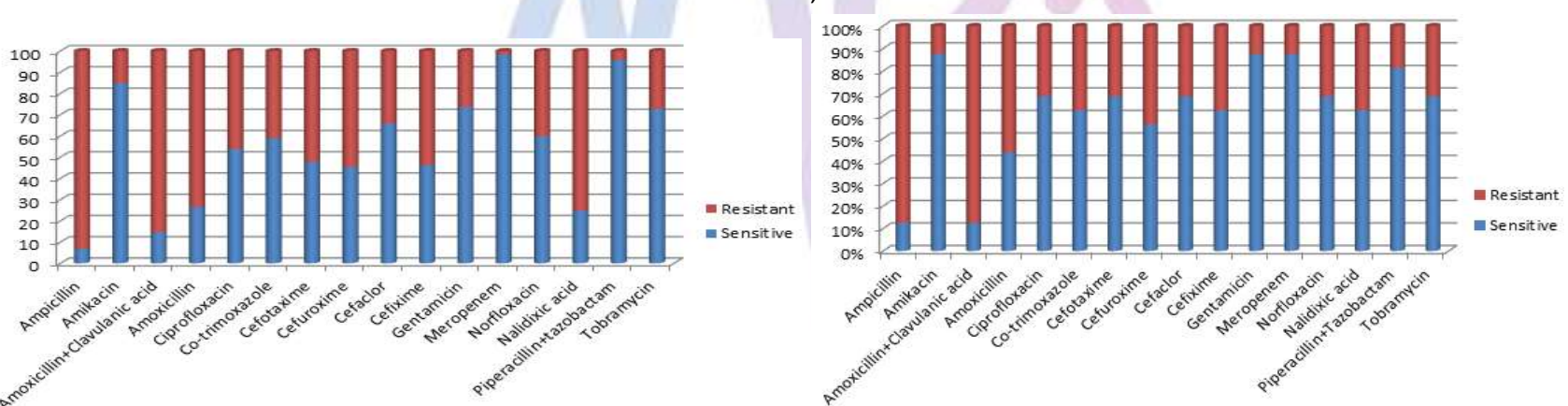

Figure 1

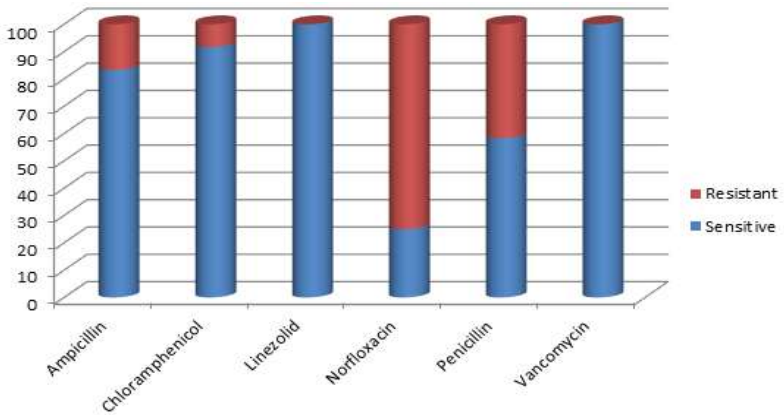

Figure 3
Figure 2

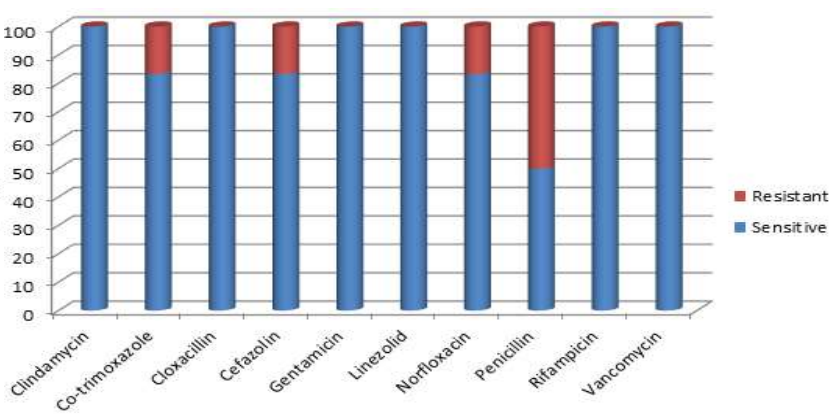

Figure 4

Figure 1: Antibiotic susceptibility pattern of Escherichia coli isolates in \% ( $n=117$ ) Figure 2: Antibiotic susceptibility pattern of Klebsiella spp. isolates in \% ( $n=16)$; Figure 3: Antibiotic susceptibility pattern of Enterococcus spp. isolates in \% ( $n=12$ ); Figure 4: Antibiotic susceptibility pattern of Staphylococcus aureus isolates in \% $(n=6)$ 


\section{DISCUSSION}

Our study revealed an isolation rate of $16.8 \%$ (168 isolates) from the total 1000 suspected UTI cases (single organism from each of positive case). This observation is very close to another study from Jaipur, India, which reported 17.19\% isolation rate ${ }^{18}$. The predominance of female UTI cases (76.2\%) over the male $(23.8 \%)$, out of the total 168 confirmed UTI cases in this study, more or less correlates with another study from Odisha, India, which recorded $78.8 \%$ of female UTI cases and only $21.1 \%$ of male cases 19 . The age group of $>70$ in male, accounted for the most number of UTI cases (32.5\%) and among female, 21-30 age group recorded the maximum number of UTI cases (21\%), followed by $31-40$ age group (17.9\%), in our study. This is in accordance with the study from Odisha, India ${ }^{19}$, where they reported that 18-27 age group in female, possessed the highest number of UTI cases $(29.2 \%)$, followed by 28-37 group (26.2\%). Also, among male, $>68$ age group possessed the majority of the isolates (41.8\%). This observation strengthens the previous documentations, that the fertility age group of 21-40 females are the most infected UTI cases, and the elderly immuno-depleted, $>70$ males are the mostly infected UTI group. Out of the confirmed UTI cases, $54.8 \%$ were from OPD and remaining $45.2 \%$ from IPD, in our study. This observation varies very much from another study from Nagaland, India, wherein, the authors reported a whopping $82.9 \%$ of UTI cases from OPD and a meager $17.1 \%$ from IPD ${ }^{20}$. The predominance of Escherichia coli as the most isolated species among the UTI cases (69.6\%), followed by Klebsiella spp. (9.5\%), in our study, more or less correlates with a study in Patiala, Punjab ${ }^{21}$, wherein the authors reported that $\mathrm{E}$. coli was the most predominant isolate (67\%), followed by Klebsiella spp. (14\%). The isolation rate of Citrobacter spp. (1.2\%), Proteus mirabilis (1.8\%) and Enterococcus spp. (7.1\%), in our study is more or less similar to that of a report from Bengaluru, India, wherein, the authors observed an isolation rate of, $1.3 \%, 1.8 \%$ and $9.4 \%$, respectively ${ }^{22}$. The isolation rate of Pseudomonas spp. (1.8\%) and Staphylococcus aureus (3.5\%), out of the total UTI cases in this study, varies a little bit from another study from Odisha, India, wherein, $1.6 \%$ and $4.9 \%$ respectively, was the isolation rate ${ }^{19}$. Candida spp. isolation rate of $2.9 \%$ in our study differs from other studies elsewhere in India ${ }^{20,22}$. We have also reported 4 strains (2.4\%) of Acinetobacter spp., as well, out of the 168 UTI cases.

The increasing trend of resistance pattern of Escherichia coli isolates, for tested antibiotics, as, Amoxyclav (85.5\% of isolates), Nalidixic acid (75.3\%), Amoxicillin (73.5\%), Cefuroxime (54.7\%), Cefixime (53.8\%), Cefotaxime (52.2\%), Ciprofloxacin (46.2\%) and Norfloxacin (40.2\%), was reported in our study. By comparing this observation,
Cefuroxime and Cefotaxime have slightly less percentage of resistance, as reported from a study in Jaipur, India, where it was, $66.67 \%$ and $70 \%$, respectively ${ }^{18}$. On the other hand, Amoxyclav and Nalidixic acid were showing higher levels of resistance of $80 \%$ and $94.6 \%$ respectively, in that study as do ours. Ciprofloxacin (74.8\%) was again more resistant than our study. But, the antibiotics most sensitive reported in our study, being, Meropenem, Piperacillin+tazobactam, Amikacin and Gentamicin, more or less correlates with a study in Karnataka ${ }^{23}$, there by ascertaining the earlier findings. In the case of Klebsiella spp. also, Amoxiclav (87.5\%), turned out to be the most resistant drug in our study, which was very close to a finding from Patiala, Punjab, India $(77.2 \%)^{21}$, but, it was only $63 \%$, registered in two other studies from Bhopal, India, and North West Pakistan ${ }^{24,25}$. Only, $31,3 \%$ of the strains were resistant to Ciprofloxacin, whereas, it was $73.3 \%$ in the Punjab study ${ }^{21}$. Cefuroxime and Cefixime reported $43.7 \%$ and $37.5 \%$ of the strains in our study, but, it was $76 \%$ for cephalosporins in general in that study ${ }^{21}$. Co-trimoxazole recorded $37.5 \%$ resistance in our study, which was very less compared to another study in Gwalior, India, $(76 \%),{ }^{26}$. Meropenem, Gentamicin, Amikacin and Piperacillin+Tazobactam remained the drugs of choice against Klebsiella spp. in our study. As for the 12 strains of Enterococcus spp. isolated, only Norfloxacin $(75 \%)$ and Penicillin (41.7\%) showed resistance, among the antibiotics tested, with Vancomycin, Linezolid and Chloramphenicol remained the drugs of choice. Among the 6 isolates of Staphylococcus aureus, only Penicillin showed resistance to $50 \%$ of the strains. Among the remaining species isolated, which were very few in number to make a resistance impact, only out of the 4 strains of Acinetobacter spp., isolated all 4 were resistant to Ampicillin, Amikacin, Amoxiclav, Amoxicillin, Cefotaxime and Cefixime. There was not much of resistance recorded among the other species. Apart from all these increased pattern of antibiotic resistance among E. coli and Klebsiella spp., especially, Amoxiclav, Ciprofloxacin and cephalosporins in particular, the matter of concern was the presence of 7 multi-drug resistant strains, 4 of E. coli, 2 of Klebsiella spp., and 1 of Acinetobacter sp. As a result, a periodic elucidation of antibiotic susceptibility pattern of all the urinary pathogens is a timely requirement, at least in this part of South Trivandrm district of Kerala, India, as there is no report of substance, like our study available, so far.

\section{CONCLUSIONS}

Escherichia coli being the predominant isolate, showed the highest level of resistance to Ampicillin (93.2\%), followed by Amoxiclav (85.5\%), Nalidixic acid (75.3\%), Amoxicillin (73.5\%), Cefuroxime (54.7\%), Cefixime 
(53.8\%), Cefotaxime (52.2\%), Ciprofloxacin (46.2\%) and Norfloxacin (40.2\%). Among Klebsiella spp., Ampicillin and Amoxiclav were the most resistant antibiotics $(87.5 \%)$, followed by Amoxicillin (56.3\%), Cefuroxime (43.7\%) and Cefixime and Co-trimoxazole, $37.5 \%$ each. As for the 12 strains of Enterococcus spp. isolated, only Norfloxacin $(75 \%)$ and Penicillin $(41.7 \%)$ showed resistance, among the antibiotics tested. Among the 6 isolates of Staphylococcus aureus, only Penicillin showed resistance to $50 \%$ of the strains. Among the remaining species isolated, which were very few in number to make a resistance impact, only out of the 4 strains of Acinetobacter spp., isolated, all 4 were resistant to Ampicillin, Amikacin, Amoxiclav, Amoxicillin, Cefotaxime and Cefixime. Meropenem, Piperacillin+Tazobactam, Amikacin and Gentamicin remained the most sensitive drugs, in general, to act as reserve antibiotics to be instituted, in case of severe complicated cases of UTI. Apart from all these increased pattern of antibiotic resistance among E. coli and Klebsiella spp., especially for Amoxiclav, Ciprofloxacin and cephalosporins in particular, the matter of concern was the presence of 7 multi-drug resistant strains, 4 of E. coli, 2 of Klebsiella spp., and 1 of Acinetobacter sp. As a result, constant testing of uropathogens isolated then and there, for their ever changing antibiotic susceptibility pattern is the timely requirement, especially in the southern part of Trivandrum district, Kerala, India, like this study.

Acknowledgements

We are thankful to Dr. S Rema Devi, Professor and Head of the department of Microbiology, Dr. Somervell Memorial CSI Medical College and Hospital, Karakonam, Trivandrum dist., Kerala, India, for permitting me to carry out this work. Also, we are indebted to the Director and the Principal of our institution for their constant encouragement in research related activities.

\section{REFERENCES}

1. Ronald AR, Nicolle LE, Stamm E, Krieger J, Warren J, Schaeffer A, et al.. Urinary tract infection in adults: Research priorities and strategies. Int J Antimicrob Agents 2001;17:343-8.

2. De Backer D, Christiaens T, Heytens S, de Sutter A, Stobberingh EE, Verschraegen G. Evolution of bacterial susceptibility pattern of Escherichia coli in uncomplicated urinary tract infections in a country with high antibiotic consumption: A comparison of two surveys with a 10 year interval. J Antimicrob Chemother 2008;62:364-8.

3. Bishop BL, Duncan MJ, Song J, Li G, Zaas D, Abraham SN. Cyclic AMP-regulated exocytosis of Escherichia coli from infected bladder epithelial cells. Nat Med. 2007;13:625-30.

4. Dimitrov TS, Udo EE, Emara M, Awni F, Passadilla R. Etiology and antibiotic susceptibility patterns of community acquired urinary tract infections in a Kuwait Hospital. Med Princ Pract 2004;13:334-9.
5. Cryz SJ Jr, Fürer E, Germanier R. Protection against fatal Klebsiella pneumoniae burn wound sepsis by passive transfer of anticapsular polysaccharide. Infect Immun 1984;45:139-42.

6. Manikandan C, Amsath A. Antibiotic susceptibility pattern of Klebsiella pneumoniae isolated from urine samples. Int J Curr Microbiol Appl Sci 2013;2:330-7.

7. Manikandan S, Ganesapandian S, Singh M, Kumaraguru AK. Antimicrobial susceptibility pattern of urinary tract infection causing human pathogenic bacteria. Asian J Med Sci 2011;3:56-60.

8. Humayun T, Iqbal A. The culture and sensitivity pattern of urinary tract infections in females of reproductive age group. Ann Pak Inst Med Sci 2012;8:19-22.

9. Afzal S, Naemullah S. Spectrum of the microorganisms in children with urinary tract infection. J Rawalpindi Med Coll 2008;12:44-6.

10. Ramanath KV, Shafiya SB. Prescription pattern of antibiotic usage for urinary tract

infection treated in a rural tertiary care hospital. Indian $\mathrm{J}$ Pharm Pract 2011;4:57-63.

11. Akram M, Shahid M, Khan AU. Etiology and antibiotic resistance patterns of community acquired urinary tract infections in JNMC Hospital, Aligarh, India. Ann Clin Microbiol Antimicrob 2007;6:6.

12. Kothari A, Sagar V. Antibiotic resistance in pathogens causing community acquired urinary tract infections in India: A multicentric study. J Infect Dev Ctries 2008;2:354-8.

13. Biswas D, Gupta P, Prasad R, Sinha V, Arya M, Kumar A. Choice of antibiotic for empirical therapy of acute cystitis in setting of high antimicrobial resistance. Indian J Med Sci 2006;60:53-8.

14. Dilip Chandrasekhar, Athira Dollychan, Bincy Mary Roy, Shinu Cholamughath and Jaffer Chalil Parambil. Prevalence and antibiotic utilization pattern of uropathogens causing community acquired urinary tract infection in Kerala, India. J of Basic Clinc Physio Pharm 2018;29(6).

15. Collee G DP, Fraser G, Marmian P. Mackey and MacCartney's Practical Medical Microbiology. 14th ed. Vol. 2. 2003. Churchill Livingstone Publishers. Longman Singapore.

16. Clinical and Laboratory Standards Institute: Performance standards for antimicrobial susceptibility testing. 21st Informational Supplement, CLSI-2012. M100-S21, Vol.31 No.1. Wayne, PA: Clinical and Laboratory Standards Institute.

17. National Committee for Clinical Laboratory Standards. Performance standards for antimicrobial disk susceptibility tests. Approved standard M2 A7 NCCLS, Villanova, PA: 1995. p. 15.

18. Smita Sood, Ravi Gupta. Antibiotic resistance pattern of community acquired uropathogens at a tertiary care hospital in Jaipur, Rajasthan. Ind $\mathrm{J}$ Com Med. 2012;37(1):39-44.

19. Muktikesh Dash, Sanghamitra Padhi, Indrani Mohanty, Pritilata Panda, Banojini Parida. Antimicrobial resistance in pathogens causing urinary tract infections in a rural community of Odisha, India. J Family Com Med. $2013 ; 20(1): 20-26$. 
20. Sedevi Angami, Nungsangmeren Jamir, Pratap Chandra Sarma, Akshay Chandra Deka. Urinary infection, its causative organism, and antibiotic susceptibility in Nagaland. Arch Med Health Sci. 2015;3(1):40-43.

21. Neetu Sharma, Anita K Gupta, Geeta Walia, Rupinder Bakhshi. A retrospective study of of the changing trends of antimicrobial resistance of Klebsiella pneumoniae isolated from urine samples over last 3 years (2012-2014). J Nat Sci Bio Med. 2016;7(1):39-42.

22. Kalal BS, Nagaraj S. Urinary tract infections: a retrospective, descriptive study of causative organisms and antimicrobial pattern of samples received for culture, from a tertiary care setting. GERMS 2016;6(4):132-138.

23. Kulkarni SR, Peerapur BV, Sailesh KS. Isolation and antibiotic susceptibility pattern of Escherichia coli from urinary tract infections in a tertiary care hospital of North Eastern Karnataka. J Nat Sc Biol Med 2017;8:176-180.

24. Kumar AR, Kalpana S. Prevalence and antimicrobial susceptibility pattern of Klebsiella pneumonia causing urinary tract infection and issues related to the rational selection of antimicrobials. Sch J Appl Med Sci 2013;1:395-9.

25. Ullah F, Malik SA, Ahmed J. Antimicrobial susceptibility pattern and ESBL prevalence in Klebsiella pneumoniae from urinary tract infections in the North-West of Pakistan. Afr J Microbiol Res 2009;3:676- 680.

26. Sikarwar AS, Batra HV. Prevalence of antimicrobial drug resistance of Klebsiella pneumoniae in India. Int J Biosci Biochem Bioinformatics 2011;1:211-215.

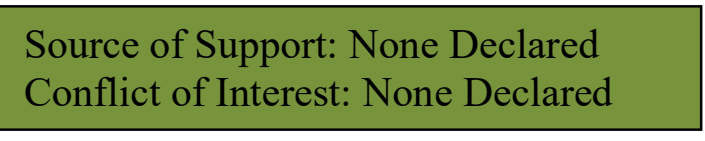

Policy for Articles with Open Access:

Authors who publish with MedPulse International Journal of Microbiology (Print ISSN: 2550-7648) (Online ISSN: 2636-4646) agree to the following terms: Authors retain copyright and grant the journal right of first publication with the work simultaneously licensed under a Creative Commons Attribution License that allows others to share the work with an acknowledgement of the work's authorship and initial publication in this journal.

Authors are permitted and encouraged to post links to their work online (e.g., in institutional repositories or on their website) prior to and during the submission process, as it can lead to productive exchanges, as well as earlier and greater citation of published work. 